\title{
O TRABALHO EDUCATIVO DO AGENTE COMUNITÁRIO DE SAÚDE NAS VISITAS DOMICILIARES EM DOIS MUNICÍPIOS DO BRASIL
}

\author{
THE EDUCATIONAL WORK OF COMMUNITY HEALTH WORKERS IN HOME VISITS IN TWO \\ MUNICIPALITIES OF BRAZIL
}

\section{EL TRABAJO EDUCATIVO DEL AGENTE COMUNITARIO DE SALUD EN LAS VISITAS DOMICILIARES EN DOS MUNICIPIOS DE BRASIL}

\author{
Dixis Figueroa Pedraza ${ }^{1}$ \\ Ana Carolina Dantas Rocha ${ }^{2}$ \\ Márcia Cristina Sales ${ }^{3}$
}

Resumo O estudo objetivou descrever e analisar possíveis diferenças nas atividades de educação em saúde realizadas por agentes comunitários de saúde durante a visita domiciliar. Foram analisados os dados de dois estudos transversais no contexto das Unidades Básicas de Saúde da Família dos municípios de Campina Grande (2010) e Queimadas (2011), no estado da Paraíba, Brasil. Em Campina Grande, foram elegíveis mulheres com crianças menores de um ano, selecionadas por sorteio sistemático, de 30\% das unidades de saúde $(\mathrm{n}=508)$. Em Queimadas, foram elegíveis todas as mulheres com crianças nascidas durante o ano de 2009, residentes no município e atendidas em todas as 16 unidades de saúde $(\mathrm{n}=204)$. As mães foram questionadas sobre as atividades educativas desenvolvidas pelos agentes durante as visitas domiciliares. Uma maior proporção de mulheres indicou não receber visitas domiciliares no município de Campina Grande, foram encontradas deficiências relacionadas a orientações sobre o cuidado com a saúde da criança nos dois municípios e maior frequência de atividades educativas entre os agentes de Queimadas. As diferenças relativas ao trabalho dos agentes comunitários de saúde podem envolver fatores como desvalorização profissional e precárias condições de trabalho. Destaca-se a importância da capacitação e preparação dos enfermeiros para a supervisão do trabalho dos agentes.

Palavras-chave agentes comunitários de saúde; Programa Saúde da Família; educação popular em saúde.
Abstract The study aimed to describe and analyze possible differences in the activities of health education performed by community health workers during a home visit. Data of two transversal studies were analyzed in the context of Family Health Units in the municipalities of Campina Grande (2010) and Queimadas (2011), in the state of Paraíba, Brazil. In Campina Grande, women whose children were younger than one year old were eligible, selected by systematic lottery, from $30 \%$ of the health units $(n=508)$. In Queimadas, all women whose children were born within the year of 2009, those who resided in the municipality and those who were treated in any of the 16 health units $(n=204)$ were eligible. The mothers were questioned about the educational activities developed by the agents during the home visits. A larger proportion of women indicated not to receive home visits in the municipality of Campina Grande, and deficiencies were found related to orientation on how to care for children's health in the two municipalities, and there was a higher frequency of educational activities in the agents from Queimadas. The differences related to the work of community health workers may involve elements such as professional devaluation and precarious work conditions. It must be emphasized the importance of the training and preparation of nurses for the supervision of the health workers.

Keywords community health workers; Family Health Program; popular education in health. 


\section{Introdução}

A atenção primária em saúde (APS) é considerada a chave na obtenção de níveis de saúde que possibilitem levar uma vida social e economicamente produtiva. Educação em saúde, saneamento básico e ambiental, programas de saúde materno-infantil, prevenção e tratamento de doenças endêmicas e comuns, medicamentos essenciais e promoção da nutrição constituem os principais componentes da APS (Vidal et al., 2003).

No Brasil, como proposta de reorientação do modelo assistencial baseado nos pressupostos da APS, o Ministério da Saúde concebeu o Programa de Agentes Comunitários de Saúde (Pacs), em 1991, e o Programa Saúde da Família (PSF), em 1994 (Baralhas e Pereira, 2011; Santos et al., 2011). Tanto o trabalho no Pacs quanto o trabalho no PSF é alicerçado na figura do agente comunitário de saúde (ACS). Trata-se de um trabalhador sui generis, com identidade comunitária, que realiza as principais ações, tarefas que não se restringem ao campo da saúde, com interface na assistência social, educação e meio ambiente (Bornstein e Stotz, 2008; Bachilli, Scavassa e Spiri, 2008; Marzari, Jungues e Selli, 2011).

O ACS, por suas características próprias de vínculo com a comunidade e com a equipe de saúde, é considerado o principal elo entre esses dois elementos, possibilita o fortalecimento do vínculo entre os profissionais de saúde e a família, e proporciona a aproximação das ações de saúde ao contexto domiciliar (Cardoso e Nascimento, 2010; Filgueiras e Silva, 2011; Santos et al., 2011). Além disso, o ACS tem papel protagonista no que se refere à relação de trocas de experiências, aos saberes populares de saúde e à identidade com a cultura, linguagem e costumes de sua própria comunidade (Costa e Ferreira, 2011; Santos et al., 2011). Acredita-se, assim, que o ACS represente um elemento essencial na reorganização dos serviços de saúde (Bezerra, Espírito Santo e Batista Filho, 2005; Cardoso e Nascimento, 2010; Peres et al., 2011). O ACS representa um recurso humano central e estratégico na implantação de ações de promoção da saúde, envolvendo as pessoas, seus conhecimentos e entornos (Santos, Pierantoni e Silva, 2010).

Contudo, ressalta-se que, apesar das funções estratégicas e complexas atribuídas ao ACS, sua formação técnica é curta, com predomínio do enfoque biologicista (Hildebrand e Shimizu, 2008). Somente em 2002 a profissão de ACS foi reconhecida, destacando-se a falta de abordagens e instrumentos adequados de preparação (Bornstein e Stotz, 2008). Estudo que analisou possibilidades e limitações do ACS revelou que as ações desenvolvidas por ele ainda não correspondem às expectativas da equipe de saúde, do governo, da comunidade e do próprio agente (Santos e Fracolli, 2010).

Para operacionalizar suas ações, os ACSs utilizam a entrevista, a visita domiciliar, o cadastramento das famílias, o mapeamento da comunidade e as 
reuniões comunitárias como instrumentos de trabalho. A visita domiciliar é considerada, além do meio mais importante de ligação entre a comunidade e os serviços de saúde, o principal instrumento de trabalho dos ACSs. Na visita, o ACS tem a oportunidade de conhecer os agravos que acometem a comunidade, comunicar aos membros da equipe do PSF a sua percepção e retornar à comunidade com atividades, principalmente educativas, que ajudem a solucionar os problemas encontrados (Araújo e Assunção, 2004; Alves e Santos, 2007; Lima, Silva e Bousso, 2010).

O presente artigo descreve e analisa possíveis diferenças nas atividades de educação em saúde realizadas por agentes comunitários de saúde durante a visita domiciliar em dois municípios da Paraíba, Brasil.

\section{Metodologia}

Estudo analítico sobre as orientações educativas recebidas por mulheres durante as visitas domiciliares de ACSs, realizado por meio da análise comparativa dos dados de dois estudos transversais realizados nos municípios de Campina Grande e Queimadas, Estado da Paraíba, Brasil. Os municípios de Campina Grande e Queimadas pertencem à Região Metropolitana de Campina Grande, inserida na unidade geoambiental do planalto da Borborema, sendo municípios limítrofes distanciados apenas em 14 km, com características geográficas similares. Em relação às condições socioeconômicas, todos os dois municípios possuem Índice de Desenvolvimento Humano (IDH) médio.

O município de Campina Grande é o segundo maior município do estado, com área de 599,6 km² e população de 376.060 habitantes. Possui um sistema de saúde composto por seis distritos sanitários com 67 Unidades Básicas de Saúde da Família (UBSs), perfazendo uma cobertura de $71 \%$ da população, e foi pioneiro na implantação do PSF em 1994. O município de Queimadas tem área de 409,2 km² e população de 41.297 habitantes. O sistema de saúde do município é constituído por 16 UBSs, perfazendo uma cobertura de 100\% da população.

No município de Campina Grande, foram elegíveis para o estudo mulheres com crianças menores de um ano, selecionadas por sorteio sistemático, de $30 \%$ das UBSs. As unidades de saúde foram selecionadas segundo alguns parâmetros de escolha: a) localizarem-se em áreas da cidade com situação socioeconômica semelhante; b) serem unidades consideradas de bom padrão relativo de atendimento; e c) apresentarem baixa rotatividade de servidores. O sorteio ocorreu de forma aleatória, considerando a divisão por distritos sanitários, com a finalidade de representar os diferentes espaços geográficos e áreas da cidade. No município de Queimadas, foram elegíveis para o estudo todas as mulheres com crianças nascidas durante o ano de 2009 (entre 18 e 30 meses), residentes no município e atendidas em todas as 16 UBSs. 
Em ambos os estudos, a coleta de dados foi realizada da mesma maneira e com a mesma equipe de trabalho de campo devidamente treinada. A coleta dos dados aconteceu no período de junho a dezembro de 2010 em Campina Grande e nos meses de julho e agosto de 2011 em Queimadas.

As informações foram coletadas, principalmente, nas UBSs ou no próprio domicílio, quando do não comparecimento das entrevistadas às unidades de saúde, por meio da aplicação de um questionário. O questionário continha questões fechadas que abordavam características das crianças, das mães, das famílias e da assistência à saúde. Nesse último item, as mulheres foram questionadas sobre as atividades educativas desenvolvidas pelos ACSs durante as visitas domiciliares. As questões foram respondidas livremente pelas entrevistadas, com alternativas de resposta sim ou não. Além disso, questionou-se sobre o tempo de intervalo das visitas (se semanal, quinzenal, mensal ou maior de um mês) e a percepção das próprias usuárias sobre a qualidade das visitas domiciliares (classificada em muito boa/boa, regular ou ruim/muito ruim), como proxy de avaliação da responsividade do serviço. Segundo a Organização Mundial da Saúde (OMS), o conceito de responsividade se refere à adequação dos serviços de saúde às expectativas dos usuários (World Health Organization, 2000).

Para cada atividade educativa, a proporção de mulheres que indicaram receber orientação na visita domiciliar do ACS no município de Campina Grande foi comparada com a do município de Queimadas. O teste z foi utilizado para verificar diferenças estatisticamente significantes. Foi considerado como nível de significância estatística o valor de $\mathrm{p}<0,05$.

Os estudos que geraram os dados apresentados neste estudo foram aprovados, antes de sua realização, pelo Comitê de Ética em Pesquisa da Universidade Estadual da Paraíba: 1) Atenção básica à saúde no município de Campina Grande, Paraíba: avaliação do desempenho e efetividade da Estratégia Saúde da Família e da incorporação das ações de alimentação e nutrição, protocolada sob o número 0355013300009; 2) Situação alimentar, nutricional e atenção à saúde materno-infantil na cidade de Queimadas, Paraíba, protocolada sob o número 0170013300011 . Todos os participantes leram e assinaram o termo de consentimento livre e esclarecido.

\section{Resultados}

Foram entrevistadas 508 mulheres no município de Campina Grande e 204 no município de Queimadas. Um total de 110 entrevistadas respondeu não receber visita de ACSs, sendo 104 em Campina Grande e seis em Queimadas. Assim, a análise das atividades de educação em saúde desenvolvidas pelos ACSs durante a visita domiciliar foi realizada para 404 mulheres residentes 
no município de Campina Grande e 198 residentes no município de Queimadas. Considerando-se todas as entrevistadas, ou seja, inclusive as que responderam não receber visita do agente, a frequência de visitas domiciliares em intervalos maiores de um mês foi de 10,8\% em Queimadas e de 23,1\% em Campina Grande.

A Tabela 1 mostra que as respostas apresentadas pelas mulheres em relação às atividades educativas desenvolvidas pelos agentes apresentam diferenças, com maior frequência para muitas delas entre os ACSs do município de Queimadas. A vacinação da criança resultou a atividade educativa mais realizada nos dois municípios, sem diferença entre os mesmos, e as atividades relacionadas com o crescimento, cuidado dos dentes, consumo de alimentos ricos em ferro e tratamento/prevenção da anemia nas crianças mostraram proporções baixas, sem diferença importante ao comparar as respostas em Queimadas e Campina Grande.

Tabela 1

\begin{tabular}{|c|c|c|c|c|c|}
\hline \multirow[t]{2}{*}{ Atividades } & \multicolumn{2}{|c|}{$\begin{array}{l}\text { Campina Grande } \\
\qquad(\mathrm{N}=404)\end{array}$} & \multicolumn{2}{|c|}{$\begin{array}{l}\text { Queimadas } \\
(N=198)\end{array}$} & \multirow{2}{*}{$\begin{array}{l}\text { valor } \\
\text { de } p\end{array}$} \\
\hline & $\mathrm{n}$ & $\%$ & $\mathrm{n}$ & $\%$ & \\
\hline Crescimento da criança & 198 & 49,0 & 108 & 54,5 & 0,117 \\
\hline Vacinação da criança & 345 & 85,4 & 179 & 90,4 & 0,054 \\
\hline Alimentação da criança/aleitamento materno & 235 & 58,1 & 131 & 66,3 & 0,03 \\
\hline Cuidado dos dentes da criança & 179 & 44,3 & 102 & 51,5 & 0,057 \\
\hline Diarreia & 181 & 44,8 & 118 & 59,6 & $<0,001$ \\
\hline Infecção respiratória & 126 & 31,2 & 76 & 38,4 & 0,048 \\
\hline Tratamento da água & 194 & 48,1 & 123 & 62,1 & 0,001 \\
\hline Destino dos dejetos & 127 & 31,4 & 92 & 46,5 & $<0,001$ \\
\hline Alimentos ricos em ferro & 198 & 49,1 & 101 & 51,0 & 0,365 \\
\hline Tratamento/prevenção da anemia & 167 & 41,3 & 92 & 46,5 & 0,134 \\
\hline Suplementação com vitamina A & 160 & 39,6 & 98 & 49,5 & 0,013 \\
\hline
\end{tabular}

Fonte: As autoras.

Essa mesma tabela mostra, ainda, que a alimentação da criança/aleitamento materno foi a segunda atividade educativa mais recorrente, tanto em Queimadas quanto em Campina Grande, porém a frequência de mulheres com orientações nas visitas dos agentes do município de Queimadas foi 
maior do que em Campina Grande. Diferenças entre os grupos também foram encontradas em relação às orientações sobre diarreia, infecção respiratória, tratamento da água, destino dos dejetos e suplementação com vitamina A, com menores frequências em Campina Grande. As atividades relacionadas com a infecção respiratória e o destino dos dejetos foram as que se apresentaram mais comprometidas nos dois municípios.

A responsividade relacionada às visitas domiciliares foi similar nos dois municípios, com valores de 86,3\% para o município de Queimadas e de 83,4\% para Campina Grande, indicativos da qualidade das visitas 'boa' ou 'muito boa'. A qualidade ruim/muito ruim correspondeu a 3,1\% e 3,8\% entre as entrevistadas de Queimadas e Campina Grande, respectivamente.

\section{Discussão}

A visita domiciliar é uma das principais atividades preconizadas pelo Ministério da Saúde para o ACS (Brasil, 2001). Estudos com os próprios ACSs, em Porto Alegre/RS (Ferraz e Aerts, 2005) e Vitória/ES (Galavote et al., 2011), mostraram resultados condizentes ao indicar a prioridade que a visita domiciliar tem para eles. Entretanto, o não acompanhamento de todas as famílias sob responsabilidade do agente foi verificado em outros estudos (Ferraz e Aerts, 2005; Costa et al., 2013), bem como opiniões diferentes foram constatadas segundo a percepção de usuários de Botucatu/SP (Lima, Silva e Bousso, 2010) e São José do Norte/RS (Alves e Santos, 2007). Número de famílias muito elevado e sobrecarga de atividades podem ser fatores relacionados à realização ou não da visita domiciliar (Lima, Silva e Bousso, 2010). Em consonância com a insuficiência relacionada à visita domiciliar dos ACSs percebida pela população, os resultados do presente estudo no município de Campina Grande indicaram que uma grande quantidade de famílias não recebeu visita do ACS e parcela importante também não recebeu visita domiciliar no mínimo mensalmente.

Estudo de intervenção comunitária realizado com mulheres gestantes residentes na periferia da cidade de Rio Grande/RS mostrou que as gestantes visitadas pelos ACSs apresentaram melhores indicadores de assistência pré-natal, uma vez que deram início ao pré-natal mais precocemente e realizaram um maior número de consultas. O impacto, segundo os autores, pode ser atribuído a características implícitas ao ACS: alta frequência de visitas domiciliares, fazer parte da equipe da unidade local de saúde, integrar equipes de saúde de fácil acesso, possibilidades veiculadas pelo agendamento de consultas para todos os membros da família. Os autores desse mesmo estudo indicam que há evidência suficiente sobre a melhora de indicadores básicos de saúde, sobretudo quando ofertados em pequena escala, 
produzida pelos cuidados em saúde em nível domiciliar. Para tal afirmação os autores exemplificam o impacto das visitas domiciliares em doenças, como as infecciosas, e em cuidados preventivos, como a imunização e a amamentação, mostrado em alguns estudos (Cesar et al., 2008). Assim, a nosso ver, a perspectiva do papel mediador do ACS entre a população e os serviços de saúde deve considerar também o impacto positivo da visita domiciliar.

Atualmente, 204 mil ACSs estão em atuação no Brasil (Costa e Ferreira, 2011). As ações desenvolvidas por esses profissionais têm sido fortemente vinculadas com as visitas domiciliares (Sakata et al., 2007) e com a educação em saúde como prática de cuidado nas visitas (Kebian e Acioli, 2011). Além disso, reconhece-se que as ações dos ACSs têm significado grandes conquistas em saúde, especialmente em municípios de baixa densidade populacional e localidades onde o acesso à atenção em saúde era restrito (Brito e Domingos Sobrinho, 2009; Filgueiras e Silva, 2011). Neste estudo, uma maior frequência no recebimento de visitas domiciliares e de atividades educativas do ACS durante as visitações foi constatada no município de menor tamanho e porte populacional (Queimadas), sendo necessário o conhecimento de outros contextos.

Algumas dificuldades têm sido mencionadas como fatores importantes para o desempenho profissional dos ACSs, inclusive para o desenvolvimento da visita domiciliar. O excesso de atribuições, o alto número de famílias a serem acompanhadas, a falta de valorização profissional, a baixa remuneração e a falta de apoio de outros profissionais da equipe foram fatores citados como obstáculos por ACSs em diferentes localidades do país (Pedrosa e Teles, 2001; Espínola e Costa, 2006; Ferraz e Aerts, 2005; Ávila, 2011; Galavote et al., 2011; Kebian e Acioli, 2014). O domínio desses fatores em municípios de maior complexidade pode, talvez, implicar maiores dificuldades no exercício do trabalho do ACS. Percebemos, ainda, que esses fatores são desfavoráveis aos desafios impostos aos ACSs, o que pode repercutir negativamente nas relações recíprocas entre os membros da equipe de saúde da família, os ACSs e as famílias, bem como na satisfação dos ACSs com o trabalho.

Do mesmo jeito que para as visitas domiciliares, as atividades de educação em saúde são priorizadas pelos ACSs (Souza, Almeida e Barbosa, 2009; Costa et al., 2013), reconhecendo-as, principalmente, como ações de orientação e prevenção (Lima et al., 2012; Gazzinelli et al., 2013), representação que pode influenciar a decisão sobre as atividades educativas a desenvolver. Problemas de conhecimento associados à falta de capacitação têm sido um ponto bastante discutido entre os fatores que comprometem o desenvolvimento do trabalho dos ACSs (Gomes et al., 2010). Estudo recente revelou diferenças, pautadas na qualificação na área de atuação e na percepção do preparo para atuar no SUS, nas proporções de ACSs que realizam atividades 
de educação em saúde em relação aos que não desenvolvem (Costa et al., 2013). Resultados similares aos do presente estudo foram achados com ACSs de Porto Alegre/RS (Ferraz e Aerts, 2005), os quais mencionaram as orientações sobre o calendário vacinal e os cuidados com recém-nascidos como as atividades educativas que mais costumam prestar às famílias.

No contexto do presente estudo, apesar da qualidade das orientações de educação em saúde não ser motivo de análise, a frequência com que um determinado tema é tratado pode ser expressão da confiança para discorrer sobre ele. Nessa conjuntura, destaca-se, considerando a necessidade de uma compreensão ampla dos problemas vivenciados pela comunidade no modelo da produção social da saúde, que o processo de capacitação dos ACSs deve compreender tanto o conhecimento específico em saúde quanto o conhecimento de estratégias educativas (Ávila, 2011; Galavote et al., 2011). A capacitação dos profissionais de saúde é outra circunstância que pode apresentar maiores dificuldades nos municípios de médio e grande porte, envolvendo questões próprias ao agente, mas também à equipe, à comunidade e ao governo.

A unanimidade sobre o papel do ACS como mediador, além de referir a mediação entre o serviço e a comunidade, destaca, também, o intermédio entre diferentes saberes (Bornstein e Stotz, 2008). Nesse contexto, às dificuldades mostradas soma-se à complexidade da educação em saúde, conforme indicam Ferraz e Aerts:

A visita domiciliar é o instrumento ideal para a educação em saúde, pois a troca de informações se dá no contexto de vida do indivíduo e de sua família. As orientações não estão prontas, pois cada casa apresenta uma realidade e é baseada nessa realidade que acontece a troca de informações (p. 353, 2005).

$\mathrm{Ou}$, conforme argumentam Brito e Domingos Sobrinho:

A educação em saúde é concebida como um processo a ser vivenciado pelo próprio ACS, que tanto precisa estar preparado para orientar e buscar qualificar-se, como conhecer o saber popular e levá-lo a interagir com o saber reificado, além de ter de considerar as condições socioeconômicas e culturais nas quais atua. Ao mesmo tempo, é uma via, não de todo consciente, que pode contribuir para as ações pela cidadania que as políticas visam implementar (p. 675, 2009).

Nesse contexto, cabe ressaltar que as práticas educativas constituem um desafio, não só para o ACS, senão para todos os profissionais da equipe. As atividades educativas são de responsabilidade também dos outros membros da equipe, que apresentam, igualmente aos ACS, problemas na formação acadêmica, um dos obstáculos para trabalhar sob a ótica da APS (Ávila, 2011). Essa situação tem repercussão especial para a área de enfermagem, 
uma vez que são estabelecidas como atribuições específicas do enfermeiro a supervisão, coordenação e realização de atividades de educação permanente dos ACSs (Brasil, 2006). Além disso, há que ressaltar a importância da atuação interdisciplinar, a qual pode estar ameaçada por problemas na interação entre enfermeiro e ACS, como tem sido constatado (Kebian e Acioli, 2014).

Ainda sobre a mediação, é meritório destacar a valorização que os usuários fazem dos ACSs, bem como a representação do valor das atividades que desenvolvem e a satisfação com o trabalho, segundo resultados de alguns estudos (Bornstein e Stotz, 2008; Lima, Silva e Bousso, 2010; Fadel, Moura e Bittencourt, 2011) em sintonia com os aqui apresentados, por meio da avaliação de responsividade, considerada adequada pelos entrevistados, pois a boa qualidade das visitas apresentou valores expressivos. Entretanto, ACSs de Teresina mostraram percepção diferente a respeito da valorização que os usuários fazem sobre o trabalho dos mesmos. Eles advertiram falta de apoio e reconhecimento, em determinadas situações, da comunidade, da própria equipe e da gestão do município (Costa e Ferreira, 2011). É importante que a expectativa colocada na figura do ACS não esteja dissociada da importância do trabalho em equipe, configurado no conceito de união e ajuda mútua (Galavote et al., 2011), para que esses profissionais possam alcançar a ansiada valorização do trabalho realizado.

Diante dos resultados obtidos, sugerem-se ações que podem potencializar o trabalho do ACS, entre elas, diminuir o número de famílias por agente; oferecer capacitações e educação permanente no Pacs e no PSF; melhorar as condições de trabalho; incentivar a valorização profissional; e melhorar a preparação dos enfermeiros para a capacitação e supervisão do trabalho dos ACSs sob sua responsabilidade. As inúmeras demandas cotidianas dos ACSs preconizam a definição urgente e precisa de competências, habilidades e saberes, bem como de ferramentas de trabalho que possam servir de subsídio para a produção do cuidado que atenda à determinação biológico-histórico-social da saúde e ressignifique o trabalho em equipe no cuidado integral.

É importante salientar que possíveis diferenças geográficas e socioeconômicas nas populações estudadas, assim como as assimetrias nos processos amostrais, são fatores que podem ter afetado os resultados das comparações realizadas. Porém, ressalta-se o estudo da percepção de usuárias de unidades de saúde com tamanhos amostrais adequados. Advertem-se, ainda, as diferenças espaciais, pensadas como sistema de objetos e ações ou de processo social, que expressam diferentes formas de organização dos serviços de saúde.

Outra possível limitação do presente estudo é a possibilidade de existência de um viés de aprovação, que ocorre principalmente quando o usuário é entrevistado no serviço. No entanto, consideramos que essa possibilidade não afeta os resultados apresentados, que evidenciam as deficiências encontradas. A falta de estudos que abordem a temática, visando, sobretudo, parâmetros de 
comparação, enfatiza o significado dos resultados encontrados e remete à necessidade de pesquisas complementares.

\section{Conflitos de interesse}

Não há.

\section{Colaboradores}

Dixis Figueroa Pedraza participou da concepção do estudo. Todos os autores participaram da análise e interpretação dos dados, redação e revisão crítica do manuscrito, aprovaram a versão final do manuscrito e declaram serem responsáveis por todos os aspectos do trabalho, garantindo sua exatidão e integridade.

Resumen El estudio objetivó describir y analizar posibles diferencias en las actividades de educación en salud realizadas por agentes comunitarios de salud durante la visita domiciliar. Fueron analizados los datos de dos estudios transversales en el contexto de las Unidades Básicas de Salud de la Familia de los municipios de Campina Grande (2010) y Queimadas (2011), en el estado de Paraíba, Brasil. En Campina Grande, fueron elegibles mujeres con niños menos de un año, seleccionadas por sorteo sistemático, de 30\% de las unidades de salud (n=508). En Queimadas, fueron elegibles todas las mujeres con niños nascidos durante el año de 2009, residentes en el municipio y atendidas en todas las 16 unidades de salud $(n=204)$. Las mamas fueron cuestionadas sobre las actividades educativas desarrolladas por los agentes durante las visitas domiciliares. Una mayor proporción de mujeres indicó no recibir visitas domiciliares en el municipio de Campina Grande, fueron encontradas deficiencias relacionadas a orientaciones sobre el cuidado con la salud del niño en los dos municipios y mayor frecuencia de actividades educativas entre los agentes de Queimadas. Las diferencias relativas al trabajo de los agentes comunitarios de salud pueden envolver factores como desvalorización profesional y precarias condiciones de trabajo. Destaca-se la importancia de la capacitación y preparación de los enfermeros para la supervisión del trabajo de los agentes.

Palabras clave agentes comunitarios de salud; Programa Salud de la Familia; educación popular en salud. 


\section{Notas}

1 Universidade Estadual da Paraíba, Programa de Pós-graduação em Saúde Pública, Campina Grande, Paraíba, Brasil.

$<$ dixisfigueroa@gmail.com>

Correspondência: Universidade Estadual da Paraíba, Mestrado em Saúde Pública, Avenida das Baraúnas, 351, Campus I, Prédio dos Mestrados, Bodocongó, CEP 58109-753, Campina Grande, Paraíba, Brasil.

2 Universidade Federal do Ceará, Programa de Pós-graduação em Enfermagem, Fortaleza, Ceará, Brasil.

$<$ aninhacdr@hotmail.com>

3 Universidade Federal do Rio Grande do Norte, Programa de Pós-graduação em Ciências da Saúde, Natal, Rio Grande do Norte, Brasil.

$<$ cristina.salles@yahoo.com.br>

\section{Referências}

ALVES, Milena P.; SANTOS, Sidney C. Um olhar sobre o trabalho dos agentes comunitários de saúde: a visita domiciliar. Revista Baiana de Enfermagem, Salvador, v. 21, n. 1, p. 71-79, 2007.

ARAÚJO, Maria R. N.; ASSUNÇÃO, Raquel S. A atuação do agente comunitário de saúde na promoção da saúde e na prevenção de doenças. Revista Brasileira de Enfermagem, Brasília, v. 57, n. 1, p. 19-25, 2004.

ÁVILA, Maria M. M. O Programa de Agentes Comunitários de Saúde no Ceará: o caso de Uruburetama. Ciência \& Saúde Coletiva, Rio de Janeiro, v. 16, n. 1, p. 349-360, 2011.

BACHILLI, Rosane G.; SCAVASSA, Ailton J.; SPIRI, Wilza C. A identidade do agente comunitário de saúde: uma abordagem fenomenológica. Ciência \& Saúde Coletiva, Rio de Janeiro, v. 13, n. 1, p. 51-60, 2008.

BARALHAS, Marilisa; PEREIRA, Maria A. O. Concepções dos agentes comunitários de saúde sobre suas práticas assistenciais. Physis, Rio de Janeiro, v. 21, n. 1, p. 31-46, 2011.
BEZERRA, Adriana F. B.; SANTO, Antônio C. G. E.; BATISTA FILHO, Malaquias. Concepções e práticas do agente comunitário na atenção à saúde do idoso. Revista de Saúde Pública, São Paulo, v. 39, n. 5, p. 809-815, 2005.

BORNSTEIN, Vera J.; STOTZ, Eduardo M. Concepções que integram a formação e o processo de trabalho dos agentes comunitários de saúde: uma revisão da literatura. Ciência \& Saúde Coletiva, Rio de Janeiro, v. 13, n. 1, p. 259-268, 2008.

BRASIL. Ministério da Saúde. Programa Agentes Comunitários de Saúde: PACS. Brasília: Ministério da Saúde, 2001.

BRASIL. Portaria n. 648/GM, de 28 de março de 2006. Aprova a Política Nacional de Atenção Básica, estabelecendo a revisão de diretrizes e normas para a organização da Atenção Básica para o Programa Saúde da Família (PSF) e o Programa Agentes Comunitários de Saúde (PACS). Diário Oficial da União, Brasília, DF, 29 mar. 2006. n. 61.

BRITO, Suerde M. O.; DOMINGOS SOBRINHO, Moisés. Os sentidos da educação em saúde 
para agentes comunitários de saúde. Revista Gaúcha de Enfermagem, Porto Alegre, v. 30, n. 4, p. 669-676, 2009.

CARDOSO, Andréia S.; NASCIMENTO, Marilene C. Comunicação no Programa Saúde da Família: o agente de saúde como elo integrador entre a equipe e a comunidade. Ciência \& Saúde Coletiva, Rio de Janeiro, v. 15, supl. 1, p. 1509-1520, 2010.

CESAR, Juraci A. et al. Diferentes estratégias de visita domiciliar e seus efeitos sobre a assistência pré-natal no extremo Sul do Brasil. Cadernos de Saúde Pública, Rio de Janeiro, v. 24, n. 11, p. 2614-2622, 2008.

COSTA, Elaine M.; FERREIRA, Danyege L. A. Percepções e motivações de agentes comunitários de saúde sobre o processo de trabalho em Teresina, Piauí. Trabalho, Educação e Saúde, Rio de Janeiro, v. 9, n. 3, p. 461-478, 2011.

COSTA, Simone M. et al. Agente Comunitário de Saúde: elemento nuclear das ações em saúde. Ciência \& Saúde Coletiva, Rio de Janeiro, v. 18, n. 7, p. 2147-2156, 2013.

ESPÍNOLA, Fabrício D. S.; COSTA, Iris C. C. Agentes comunitários de saúde do PACS e PSF: uma análise de sua vivência profissional. Revista de Odontologia da Universidade Cidade de São Paulo, São Paulo, v. 18, n. 1, p. 43-51, 2006.

FADEL, Cristina B.; MOURA, Amanda M. G.; BITTENCOURT, Monise E. Visitas domiciliares no programa de agentes comunitários de saúde: a análise de um grupo de usuários do sistema único de saúde. Revista Brasileira de Pesquisa em Saúde, Vitória, v. 13, n. 2, p. 62-67, 2011.

FERRAZ, Lucimare; AERTS, Denise R. G. C. O cotidiano de trabalho do agente comunitário de saúde no PSF em Porto Alegre. Ciência \& Saúde Coletiva, Rio de Janeiro, v. 10, n. 2, p. 347-355, 2005.

FILGUEIRAS, Andréa S.; SILVA, Ana L. A. Agente Comunitário de Saúde: um novo ator no cenário da saúde do Brasil. Physis, Rio de Janeiro, v. 21, n. 3, p. 899-915, 2011.
GALAVOTE, Heletícia S. et al. Desvendando os processos de trabalho do agente comunitário de saúde nos cenários revelados na Estratégia Saúde da Família no município de Vitória (ES, Brasil). Ciência \& Saúde Coletiva, Rio de Janeiro, v. 16, n. 1, p. 231-240, 2011.

GAZZINELLI, Maria F. C. et al. Representações sociais da educação em saúde pelos profissionais da equipe de saúde da família. Revista Trabalho, Educação e Saúde, Rio de Janeiro, v. 11, n. 3, p. 553-571, 2013.

GOMES, Karine O. et al. O Agente Comunitário de Saúde e a consolidação do Sistema Único de Saúde: reflexões contemporâneas. Physis, Rio de Janeiro, v. 20, n. 4, p. 1143-1164, 2010.

HILDEBRAND, Stella M.; SHIMIZU, Helena E. Percepção do agente comunitário sobre o Programa Família Saudável. Revista Brasileira de Enfermagem, Brasília, v. 61, n. 3, p. 319-324, 2008.

KEBIAN, Luciana V. A.; ACIOLI, Sonia. A visita domiciliar de enfermeiros e agentes comunitários de saúde da Estratégia Saúde da Família. Revista Eletrônica de Enfermagem, Goiânia, v. 16, n. 1, p. 161-169, 2014.

KEBIAN, Luciana V. A.; ACIOLI, Sonia. Visita domiciliar: espaço de práticas de cuidado do enfermeiro e do agente comunitário de saúde. Revista Enfermagem UERJ, Rio de Janeiro, v. 19, n. 3, p. 403-409, 2011.

LIMA, Ariane N.; SILVA, Lucia; BOUSSO, Regina S. A Visita Domiciliária realizada pelo agente comunitário de saúde sob a ótica de adultos e idosos. Saúde e Sociedade, São Paulo, v. 19, n. 4, p. 889-897, 2010.

LIMA, Christiano A.B. et al. Representações sociais sobre educação em saúde de agentes comunitários: pistas para educação permanente. Cogitare Enfermagem, Curitiba, v. 17, n. 1, p. 15-20, 2012.

MARZARI, Carla K.; JUNGUES, José R.; SELLI, Lucilda. Agentes comunitários de saúde: perfil e formação. Ciência \& Saúde Coletiva, Rio de Janeiro, v. 16, supl. 1, p. 120-135, 2011. 
PEDROSA, José I. S.; TELES, João B. M. Consenso e diferenças em equipes do programa saúde da família. Revista de Saúde Pública, São Paulo, v. 35, n. 3, p. 303-311, 2001.

PERES, Cássia R. F. B. et al. O agente comunitário de saúde frente ao processo de trabalho em equipe: facilidades e dificuldades. Revista da Escola de Enfermagem da USP, São Paulo, v. 45, n. 4, p. 905-911, 2011.

SAKATA, Karen N. et al. Concepções da equipe de saúde da família sobre as visitas domiciliares. Revista Brasileira de Enfermagem, Brasília, v. 60, n. 6, p. 659-664, 2007.

SANTOS, Karina T. et al. Agente comunitário de saúde: perfil adequado a realidade do Programa Saúde da Família?. Ciência \& Saúde Coletiva, Rio de Janeiro, v. 16, supl. 1, p. 1023-1028, 2011.

SANTOS, Luciana P. G. S; FRACOLLI, Lislaine A. O Agente Comunitário de Saúde: possibilidades e limites para a promoção da saúde. Revista da Escola de Enfermagem USP, São Paulo, v. 44, n. 1, p. 76-83, 2010.
SANTOS, Maria R.; PIERANTONI, Celia R.; SILVA, Lorena L. Agentes Comunitários de Saúde: experiências e modelos do Brasil. Physis, Rio de Janeiro, v. 20, n. 4, p. 1165-1181, 2010.

SOUZA, Expedita B.; ALMEIDA, Táina B. S.; BARBOSA, Simone P. Compreensão dos agentes comunitários de saúde sobre a importância do seu papel na visita domiciliar. Revista Enfermagem Integrada, Ipatinga, v. 2, n. 2, p. 320-331, 2009.

VIDAL, Suely A. et al. Aplicação da estratégia da Atenção Integral às Doenças Prevalentes da Infância (AIDPI) por Agentes Comunitários de Saúde. Revista Brasileira de Saúde Materno Infantil, Recife, v. 3, n. 2, p. 205-214, 2003.

WORLD HEALTH ORGANIZATION. The World Health Report: Health System: Improving Performance. Genebra: World Health Organization, 2000.

Recebido em 07/02/2014

Aprovado em 19/11/2014 